\title{
MASS-LOSING AGB STARS IN THE LMC
}

\author{
JACCO TH. VAN LOON AND ALBERT A. ZIJLSTRA \\ European Southern Observatory \\ Garching bei München, Germany \\ PATRICIA A. WHITELOCK \\ South African Astronomical Observatory \\ CECILE LOUP \\ European Southern Observatory, Santiago, Chile \\ AND \\ L.B.F.M. WATERS \\ University of Amsterdam, The Netherlands
}

\begin{abstract}
We show the results of an infrared study of a sample of heavily obscured AGB stars in the LMC. Both carbon-rich and oxygen-rich masslosing AGB stars can be found at both high and low luminosities, but the percentage of carbon stars decreases with increasing luminosity. The optical depth of the circumstellar envelopes also decreases with increasing luminosity, while the mass-loss rates are (nearly) constant with luminosity. We also show tentative evidence for having found the first post-AGB stars in the LMC.
\end{abstract}

\section{Introduction}

When stars with ZAMS masses in the range $1-8 M_{\odot}$ evolve on the AGB, they enter a phase of thermal pulsations in the stellar interior. The convective mixing of the stellar interior that occurs during these thermal pulses can lead to a change in the carbon-to-oxygen ratio of the photosphere (the third dredge-up). In this way, the carbon-to-oxygen ratio of the photosphere can evolve from oxygen-dominated to carbon-dominated; a carbon star is formed. However, nuclear burning at the bottom of the convection mantles of the most massive AGB stars (Hot Bottom Burning) may convert carbon stars into nitrogen-overabundant S stars (see, e.g., Lattanzio in these proceedings for a discussion of the internal structure of AGB stars). 
The thermal pulsing AGB is also the phase during which stars undergo strong photospheric pulsations, triggering the heaviest mass loss that these stars ever experience. With mass-loss rates typically of the order $10^{-6}$ to $10^{-4} M_{\odot \mathrm{yr}^{-1}}$, mass loss dominates over nuclear burning, eventually terminating the star's evolution on the AGB. In the upper layers of the star's extended atmosphere dust grains form, and radiation pressure thereon drives the expelled matter away from the star. As the chemistry of the photospheres of carbon stars is carbon-dominated, so are their dusty circumstellar envelopes. This may lead to differences in the mass-loss characteristics between carbon stars and oxygen (M-type) stars (see, e.g., Fleischer et al., Sedlmayr \& Winters, and Cherchneff, all in these proceedings, for a discussion on dust formation and mass loss from AGB stars).

The evolution of AGB stars can only be unravelled by studying a sample of stars with known luminosities, and covering the entire AGB. This is impossible to do in the Milky Way, but the Magellanic Clouds are perfect for it. Initially, LMC studies had been limited to optically bright AGB stars (e.g. Blanco et al. 1980; Westerlund et al. 1981), and it is only very recently that the first samples of heavily obscured AGB stars in the LMC have been compiled, based on IRAS data (Reid 1991; Wood et al. 1992; Zijlstra et al. 1996; for the SMC see the poster paper by Groenewegen \& Blommaert, these proceedings). The results of ground-based near-infrared surveys, optical and near-infrared monitoring, mm-searches for maser emission, and notably mid-infrared photometry and spectroscopy by the ISO satellite are currently yielding a gigantic increase in the amount of data on mass-losing AGB stars in the LMC, expected to result in a similarly huge leap in our knowledge of mass loss and stellar evolution on the AGB.

\section{Searching for Mass-losing AGB Stars in the LMC}

Searches for near-infrared counterparts of IRAS point sources in the LMC resulted in the first samples of infrared stars in the LMC. However, besides mass-losing AGB stars, these could also be red supergiants, foreground stars, young stellar objects, or post-AGB stars. Zijlstra et al. (1996), mainly on the basis of luminosities and infrared colors, identified the mass-losing AGB stars among them. Following upon this result, we performed groundbased $J H K L N$ photometry of these stars to derive accurate luminosities, and to estimate mass-loss rates and carbon-to-oxygen ratios from the infrared colors. In this analysis, all observations were reduced to a single epoch - viz. the epoch of the $N$-band measurement - and the sample will accordingly be referred to as the Nov94 sample.

We have attempted to extend the known sample of mass-losing AGB stars in the LMC by continuing the search for near-infrared counterparts of 
remaining IRAS point sources. Preliminary results of this sample extension (JAN96) are included in the present paper.

\section{Identifying Mass-Losing Carbon and Oxygen AGB Stars}

Guglielmo et al. (1993) showed that carbon and oxygen stars may be separated in a near/mid-infrared color-color diagram. Zijlstra et al. (1996) showed that in this way, a $K-[12]$ versus $H-K$ diagram can be used to distinguish carbon from oxygen stars. A $K-[12]$ versus $J-K$ diagram works even better, but it is more difficult to go sufficiently deep at $J$ to actually detect the most obscured AGB stars.

We show as Figure 1 the $K-[12]$ versus $J-K$ diagram for the LMC stars of the NOv94 (squares) and JAN96 (solid circles) samples. The sequences of AGB stars in the Milky Way as used by Guglielmo et al. and Zijlstra et al. are given for carbon stars (dashed curve) and oxygen stars (dotted curve). The deviation of the oxygen sequence from a straight line is a consequence of the behavior of the $9.8 \mu \mathrm{m}$ silicate feature in the circumstellar envelopes of these stars. It is clear that in some parts of the diagram carbon stars and oxygen stars are well separated, while in other parts there remains ambiguity. We can nevertheless take a semi-statistical approach in classifying stars as probably oxygen-rich, probably carbon-rich, or ambiguous.

\section{Possible Post-AGB Stars in the LMC}

In the JAN96 sample, a cluster of at least six stars have a location in the $K-[12]$ versus $J-K$ diagram which is not consistent with either the carbonstar or the oxygen-star sequence. We argue that these stars may in fact be the first post-AGB stars in the LMC to have been identified as such.

Van der Veen et al. (1989) compiled a sample of presumed post-AGB stars in the Milky Way, adding (only) a few planetary nebulae and OH/IR stars. These are plotted in the $K-[12]$ versus $J-K$ diagram as well (dots). The JAN96 cluster of deviating stars clearly falls in the region where postAGB stars are located. Furthermore, the IRAS [12]-[25] colors for these JAN96 stars are redder than for the other stars of the JAN96 and Nov94 samples. This is expected for post-AGB stars: after departure from the AGB the mass-loss rate drops dramatically, resulting in a growing inner radius of the circumstellar dust envelope and, consequently, a decreasing temperature of the circumstellar dust.

The JAN96 post-AGB candidates could perhaps also be explained as being AGB stars that have experienced a helium shell flash but have not yet recovered from the resulting drop in luminosity and mass-loss rate. Candidates for this type of star in the Milky Way may be found in Whitelock et al. (1995), although with somewhat different infrared colors than ours. 


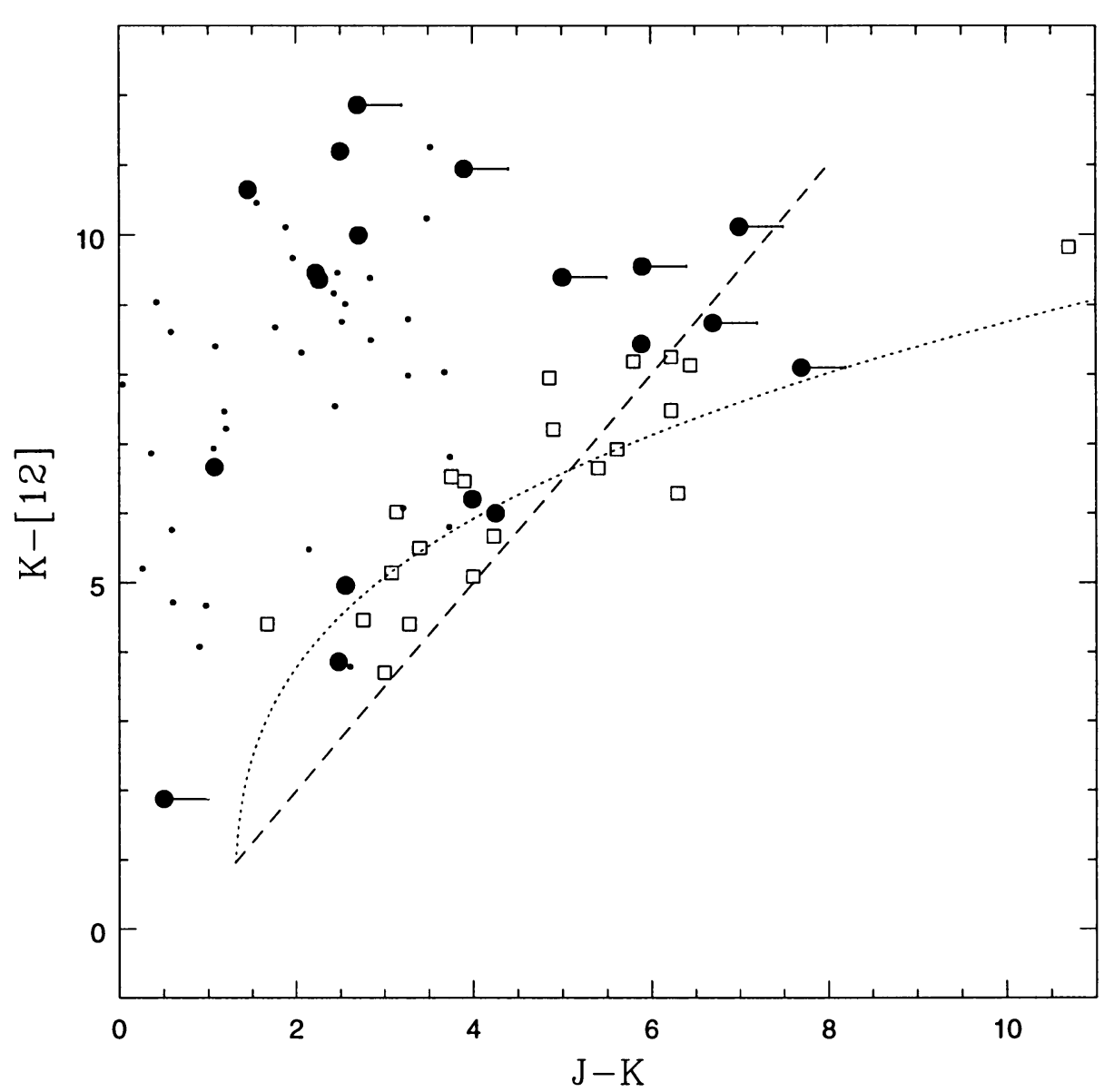

Figure 1. $K-[12]$ versus $J-K$ color-color diagram for: AGB stars in the LMC, Nov94 (squares); IR stars in the LMC, JAN96 (solid circles); and post-AGB stars in the Milky Way, van der Veen et al. 1989 (dots). Mean relations for carbon stars in the Milky Way (dashed curve) and for oxygen stars in the Milky Way (dotted curve) are also shown. Bars attached to symbols indicate non-detection at $J$, i.e. lower limits to $J-K$.

\section{Luminosities}

Obscured AGB stars emit predominantly at near- and mid-infrared wavelengths. Obscured supergiants can be erroneously classified as normal AGB stars from optical and $I$-band data if infrared photometry is not available. The sensitivity of the IRAS satellite in the mid-infrared was near the limit needed for observing obscured AGB stars at the distance of the LMC. In the mid-infrared - and even more so in the near-infrared - these stars 
are highly variable, with amplitudes often exceeding one magnitude. For the Nov94 sample, the ground-based $N$-band data allow the construction of single-epoch near- and mid-infrared spectral energy distributions. From these, single-epoch luminosities can be derived. For the JAN96 sample, the spectral energy distributions are much less certain.

The luminosity distributions of the carbon and oxygen stars indicate that the ratio of carbon stars to oxygen stars decreases with luminosity. However, one of the most likely carbon stars in the sample is very luminous $\left(M_{\text {bol }} \approx-6.8\right)$. On the other hand, mass-losing AGB stars that are fainter (say, $M_{\mathrm{bol}} \approx-5$ ) are definitely not all carbon stars.

The preliminary results from the JAN96 sample confirm and improve upon the results from the Nov94 sample. Furthermore, the stars that we tentatively classify as post-AGB stars are all of relatively low luminosity $\left(M_{\mathrm{bol}} \approx-5\right)$, consistent with fainter stars being both more numerous and longer living than brighter stars.

\section{Mass-Loss Rates}

Various methods exist for estimating mass-loss rates from AGB stars. However, they often disagree by an order of magnitude, and usually only an estimate for the dust mass-loss rate is obtained, the dust being only a minor constituent of the circumstellar matter.

We used two methods for estimating dust mass-loss rates from the Nov94 stars, using infrared colors: from $K-[12]$ (Jura 1986; Whitelock et al. 1994), and from $H-K$ (Le Bertre 1987; Epchtein et al. 1990). When applying both methods, agreement is only obtained for the most luminous stars. For the fainter stars the $K-[12]$ colors indicate greater mass-loss rates than the $H-K$ colors do. We explain this as a consequence of the winds from the fainter stars being optically thick, so that $K-[12]$ is not a : liable measure of the mass-loss rate (Whitelock et al. 1994). The $H-K$ colors suggest that the mass-loss rates are consistent with no luminosity dependence, or at most a very slow increase of mass-loss rate with increasing luminosity. The total, gas+dust mass-loss rates are about $1-2 \times 10^{-5} M_{\odot} \mathrm{yr}^{-1}, \therefore$ imilar to those of obscured AGB stars in the Milky Way (Whitelock et al. 1994).

The observed luminosity dependence of the optical depth of heavily obscured AGB stars is

$$
\tau \propto L^{-k}
$$

with $\mathrm{k} \approx 0.4-0.5$. This is identical to model predictions for single, pulsating AGB stars by Elitzur \& Ivezic (see their contributions in these proceedings). We show here that their formalism also applies to a sample of different stars. 


\section{Conclusions}

The heavily obscured AGB stars in the LMC are shown all to have similar, high mass-loss rates. The winds of brighter stars become optically thinner as the inner radius of their dusty circumstellar envelopes increases. The higher the luminosity, the lower the percentage of carbon stars, but carbon stars can probably exist up to the very highest luminosities on the AGB, while oxygen stars remain abundant at lower luminosities on the AGB.

Our search for new near-infrared counterparts of IRAS point sources in the LMC has resulted in more mass-losing AGB stars. We have also tentatively discovered several post-AGB stars. If true, this opens exciting new possibilities for studying the transition between AGB stars and white dwarfs.

Jacco van Loon wishes to express his sincere thanks to the IAU and the Symposium organizing committee for travel and subsistence grants, which made his attendance possible. Además agradece a Dr. Montserrat VillarMartín por las reuniones muy estimulantes, antes y después del congreso.

\section{References}

Blanco, V.M., McCarthy, M.F. \& Blanco, B. M. 1980, ApJ, 242, 938

Epchtein, N., Le Bertre, T. \& Lépine, J. R. D. 1990, $A \& A, 227,82$

Guglielmo, F., Epchtein, N., Le Bertre, T., Fouqué, P., Hron, J., Kerschbaum, F. \& Lépine, J.R. D. 1993, A\&A Supp., 99, 31

Jura, M. 1986, $A p J, 303,327$

Le Bertre, T. 1987, $A \& A, 176,107$

Reid, N. 1991, $A p J, 382,143$

van der Veen, W. E. C. J., Habing, H. J. \& Geballe, T. R. 1989, $A \& A$, 226, 108

Westerlund, B. E., Olander, N. \& Hedin, B. 1981, A\&A Supp., 43, 267

Whitelock, P., Menzies, J., Feast, M., Marang, F., Carter, B., Roberts, G., Catchpole, R. \& Charman, J. 1994, MNRAS, 267, 711

Whitelock, P., Menzies, J., Feast, M., Catchpole, R., Marang, F. \& Carter, B. 1995, MNRAS, 276, 219

Wood, P. R., Whiteoak, J. B., Hughes, S. M. G., Bessell, M.S., Gardner, F. F. \& Hyland, A.R. 1992, $A p J, 397,552$

Zijlstra, A. A., Loup, C., Waters, L. B. F. M., Whitelock, P. A., van Loon, J. Th. \& Guglielmo, F. 1996, MNRAS, 279, 32

\section{Discussion}

Linsky: How does the wind velocity enter into the mass-loss rate equation, and what value of the velocity is assumed?

van Loon: The dust mass-loss rate as derived from the optical depth at $1 \mu \mathrm{m}$ (which we estimate from the $H-K$ color) is a linear function of the expansion velocity of the dust envelope. We assume $10 \mathrm{~km} \mathrm{~s}^{-1}$. On the 
AGB we do not expect expansion velocities that differ by more than some $50 \%$ from this assumed value.

Feast: Presumably it is necessary to know whether the star is C- or O-rich before one can derive a mass-loss rate from, for example, an $H-K$ color, since the opacities of carbon-rich and oxygen-rich grains are different.

van Loon: That is absolutely true. The mass-loss rate we obtain from the $H-K$ color is assuming that it is a carbon star, just as many of the other methods for the determination of mass-loss rates have been designed for carbon stars. We get good estimates for the optical depth of the envelope at $1 \mu \mathrm{m}$ for our stars, but to go from these optical depths to mass-loss rates is in principle different for oxygen- and carbon-rich stars.

Sedlmayr: Similar theoretical relations connecting the parametric behavior of basic quantities (like $\tau$ vs. $v_{\text {exp }}$ ) have been derived by Dominik some years ago. How do your empirical relations fit to these theoretical findings?

van Loon: Unfortunately it is very difficult to obtain all relevant stellar parameters for any sample of AGB stars. For the LMC we have the advantage of being able to obtain absolute luminosities, which are crucial for deriving many other stellar parameters from observable quantities. We are just beginning to explore the parameter space for the mass-losing AGB stars in the LMC. Therefore we can test very global and direct relationships between different stellar parameters, but to test more detailed and complex relationships is difficult at present. We are working on it, though.

Hron: With your multiband photometry (including $12 \mu \mathrm{m}$ ) you could try to fit the energy distributions by two blackbodies to separate $\mathrm{C}$ and $\mathrm{M}$ stars and estimate mass-loss rates. This technique has been applied successfully to field stars by Kerschbaum et al. (see their two poster abstracts in these Proceedings).

van Loon: In general the spectral energy distribution is not well represented by the superposition of two blackbodies. Sometimes it is not too bad a fit to the data, but then the physical parameters derived from the blackbodies still may not always be accurate, and they can even be quite different from the real physical parameters. This method should definitely be calibrated by comparison with the physical parameters found by more correct methods, before being applied. That Kerschbaum et al. show it to work for their sample might be the result of the fact that their sample is limited to AGB stars with fairly moderate mass-loss rates: they are not very much dust-enshrouded. 